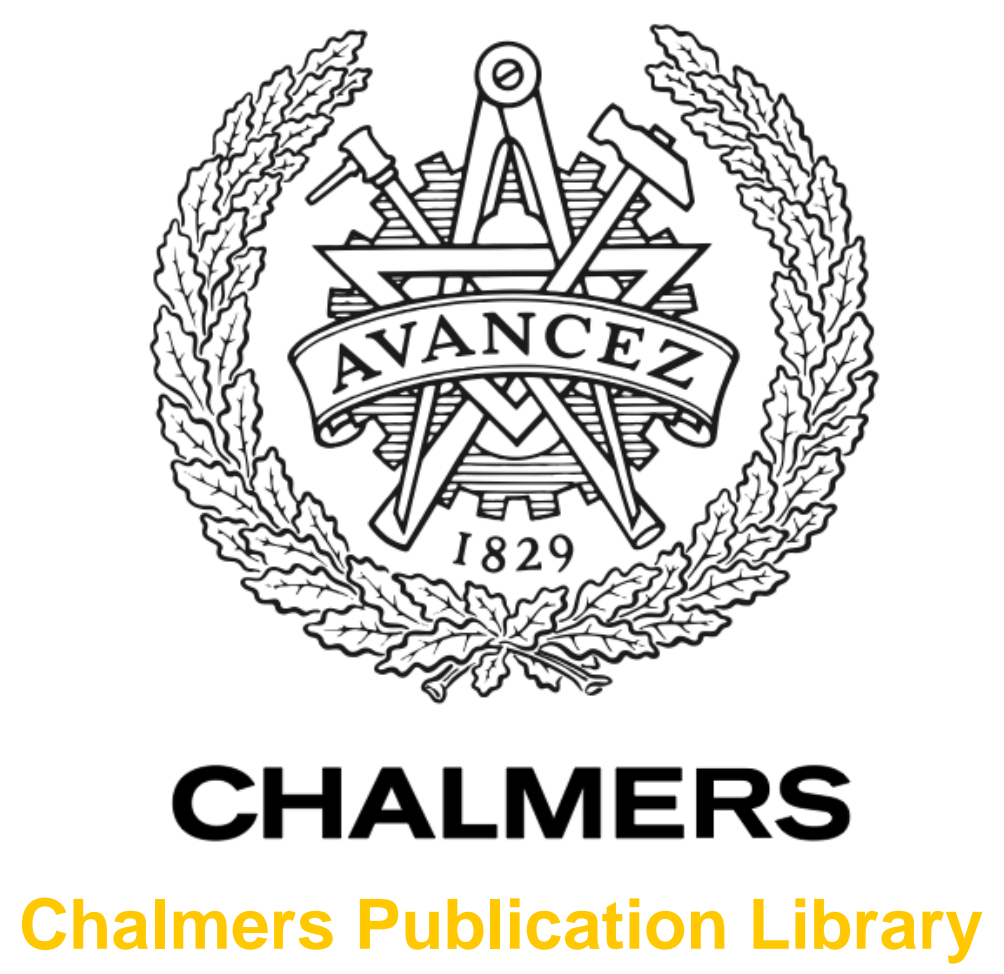

\title{
Lyapunov Function for Periodic Economic Optimizing Model Predictive Control
}

This document has been downloaded from Chalmers Publication Library (CPL). It is the author's version of a work that was accepted for publication in:

Conference on Decision and Control, 2013

Citation for the published paper:

Zanon, M. ; Gros, S. ; Diehl, M. (2013) "Lyapunov Function for Periodic Economic

Optimizing Model Predictive Control". Conference on Decision and Control, 2013

Downloaded from: http://publications.lib.chalmers.se/publication/194416

Notice: Changes introduced as a result of publishing processes such as copy-editing and formatting may not be reflected in this document. For a definitive version of this work, please refer to the published source. Please note that access to the published version might require a subscription.

Chalmers Publication Library (CPL) offers the possibility of retrieving research publications produced at Chalmers University of Technology. It covers all types of publications: articles, dissertations, licentiate theses, masters theses, conference papers, reports etc. Since 2006 it is the official tool for Chalmers official publication statistics. To ensure that Chalmers research results are disseminated as widely as possible, an Open Access Policy has been adopted.

The CPL service is administrated and maintained by Chalmers Library. 


\title{
A Lyapunov Function for Periodic Economic Optimizing Model Predictive Control
}

\author{
Mario Zanon, Sébastien Gros and Moritz Diehl
}

\begin{abstract}
Model Predictive Control (MPC) schemes are commonly using reference-tracking cost functions, which have attractive properties in terms of stability and numerical implementation. However, many control applications have clear economic objectives that can be used directly as the NMPC cost function. Such NMPC schemes are labelled Economic NMPC. Unfortunately, Economic NMPC schemes suffer from some drawbacks. In particular, stability results for economic NMPC are still very sparse. A Lyapunov function for Economic NMPC was first proposed in [1] for problems having a steady-state optimum. The present paper develops a further generalization and clarification of these results for periodic systems.

Keywords : Asymptotic stability of periodic systems, economic Model Predictive Control (MPC)
\end{abstract}

\section{INTRODUCTION}

Model Predictive Control (MPC) is an advanced feedback control technique that is gaining popularity thanks to its ability to deal with constrained, nonlinear, multiple-input multiple-output systems. Recent developments propose to include objectives having an economic nature in the cost function, rather than a more classical tracking objective penalizing the deviation of the states and inputs from a given reference. NMPC schemes that deal explicitly with economic objectives are conventionally labelled Economic NMPC (E-NMPC) in the literature. While E-NMPC allows for computing control policies that maximize the control performance explicitly, it also presents some difficulties [2], a major one being the lack of results concerning its nominal closed-loop stability.

The stability of E-NMPC via Lyapunov functions, has been first established in [1] for schemes including a terminal point constraint and a steady-state optimum, where it is assumed that a strong duality assumption holds. This work has been extended in [3] for the case of terminal cost and ellipsoidal terminal constraint. The work proposed in [4] focused on the stability of periodic systems, with some restrictions. In [5] schemes without terminal constraint nor terminal cost are considered and stability results are provided for problems exhibiting an optimal steady-state.

This paper extends the results presented in [1] to problems having optimal periodic trajectories. A rotated cost is proposed, based on which a Lyapunov function is defined. and tested for two numerical examples. As opposed to the work presented in [4], the proposed analysis does not rely on a transformed system to prove stability and applies to a more general class of systems.

M. Zanon, S. Gros and M. Diehl are with the Optimization in Engineering Center (OPTEC), K.U. Leuven, Kasteelpark Arenberg 10, B-3001 LeuvenHeverlee, Belgium. mario.zanon@esat.kuleuven.be

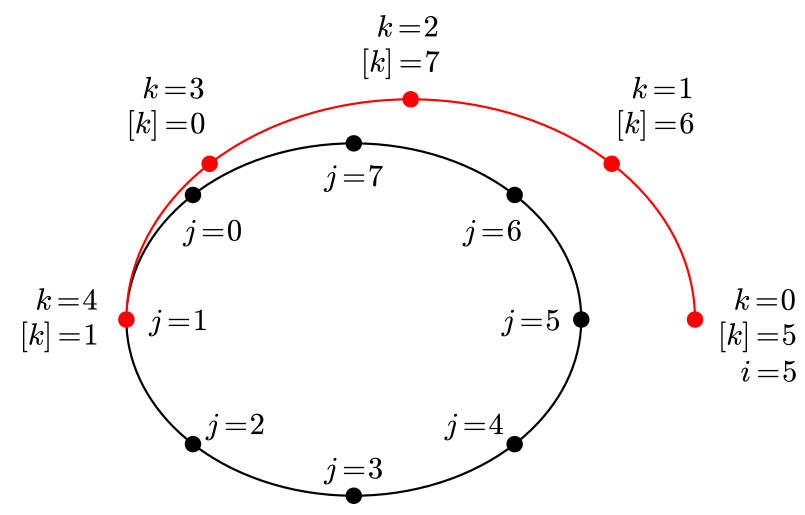

Fig. 1. Schematics of the indices at time index $i=5$ for $P=8$ and $N=4$. The optimal periodic trajectory is displayed in black and is indexed by time index $j$. The MPC trajectory is displayed in red and is indexed by time index $k$. The variables of the optimal periodic trajectory are referenced by the time index $[k]$, computed as in (1).

This paper is structured as follows. In Section II the periodic optimal control problem is defined and the economic MPC problem is formulated in Section III. Section IV proposes a new definition of the rotated cost for which stability of the MPC scheme can be shown under a hypothesis of strong duality. Two examples are provided in Section $\mathrm{V}$ and conclusions in Section VI.

\section{A. Notation}

To improve readability, in the following, the time index $j$ will be used when referring to the periodic optimal control problem while time index $k$ will be used when referring to the NMPC problem. When referring to variables relative to the periodic solution within the NMPC context, the following notation will be used

$$
[k]=i+k \quad(\bmod P), \forall k=0, \ldots, N,
$$

where $i$ is the index relative to the current time, at which the NMPC problem (3) is solved and $N$ is the NMPC prediction horizon, as defined in (3). This shifted notation allows for expressing periodic quantities as "seen" from the NMPC scheme. All indices are summarized in Table I and a schematics is given in Figure 1. See also Algorithm 1 for a more detailed explanation. 
TABLE I

LIST OF USED INDICES.

\begin{tabular}{ll}
\hline Index & Description \\
\hline$P$ & Period \\
$N$ & MPC prediction horizon \\
$i$ & Time instant at which problem $(3)$ is solved \\
$j$ & Time index relative to the periodic optimization problem \\
$k$ & Time index relative to the MPC problem \\
{$[k]$} & $i+k(\bmod P)$ \\
\hline
\end{tabular}

\section{Periodic Economic Optimization Problem}

Throughout this paper, the $P$-periodic constrained discretetime system

$$
x_{j+1}=f_{j}\left(x_{j}, u_{j}\right), \quad g_{j}\left(x_{j}, u_{j}\right) \leq 0, \quad j \geq 0,
$$

will be considered and the $P$-periodic economic stage cost function $l_{j}\left(x_{j}, u_{j}\right)$ will be used. The periodic optimization problem reads:

$$
\begin{array}{rll}
\min _{x_{0}, u_{0}, \ldots, x_{P-1}} & \sum_{j=0}^{P-1} l_{j}\left(x_{j}, u_{j}\right) & \\
\text { s.t. } & x_{j+1}=f_{j}\left(x_{j}, u_{j}\right), & \\
& x_{0}=f_{0}\left(x_{P-1}, u_{P-1}\right) & \\
& g_{j}\left(x_{j}, u_{j}\right) \leq 0, \quad j=0, \ldots, P-2
\end{array}
$$

Let the solution to problem (2) be $\left(x_{j}^{\mathrm{p}}, u_{j}^{\mathrm{p}}\right), j=0, \ldots, P-1$, which is assumed to be unique throughout the paper. Let the multipliers $\lambda_{j+1}, j=0, \ldots, P-2$ be associated with constraints (2b) and the multiplier $\lambda_{0}$ be associated with constraint (2c).

Note that, in contrast to tracking NMPC schemes, in general the functions $l_{j}\left(x_{j}, u_{j}\right)$ do not have their minimum at the solution of problem (2).

\section{ECONOMIC MPC FOR PERIODIC SYSTEMS}

Let the current state be denoted by $\bar{x}_{i}$. The proposed economic NMPC formulation solves, for each $\bar{x}_{i}$, the following optimal control problem

$$
\begin{array}{rll}
V_{N}^{[0]}\left(\bar{x}_{i}\right):= & \\
\min _{x_{0}, u_{0}, \ldots, x_{N}} & \sum_{k=0}^{N-1} l_{[k]}\left(x_{k}, u_{k}\right) & \\
\text { s.t. } \quad & x_{0}-\bar{x}_{i}=0 & \\
& x_{k+1}-f_{[k]}\left(x_{k}, u_{k}\right)=0, \quad k=0, \ldots, N-1 \\
& g_{[k]}\left(x_{k}, u_{k}\right) \leq 0, & \\
& x_{N}-x_{[N]}^{\mathrm{p}}=0, &
\end{array}
$$

where the time index $[k]$ is consistent with the definition given in (1) and does thus implicitly depend on $i$. In particular, $[0]=i(\bmod P)$ holds.

The prediction horizon $N$ is chosen independently of the period $P$. This formulation does not include any periodicity condition. Instead, the terminal constraint (3e) is imposed, such that the terminal state lies on the periodic trajectory $\left(x_{j}^{\mathrm{p}}, u_{j}^{\mathrm{p}}\right)$, solution of (2). Let the solution of (3) be denoted by $\left(x_{0}^{\star}, u_{0}^{\star}, \ldots, x_{N}^{\star}\right)$ and the corresponding E-NMPC feedback law be denoted by $u_{\mathrm{e}}^{i}\left(\bar{x}_{i}\right)=u_{0}^{\star}$. An algorithm for the implementation of this MPC scheme is provided in Algorithm 1.

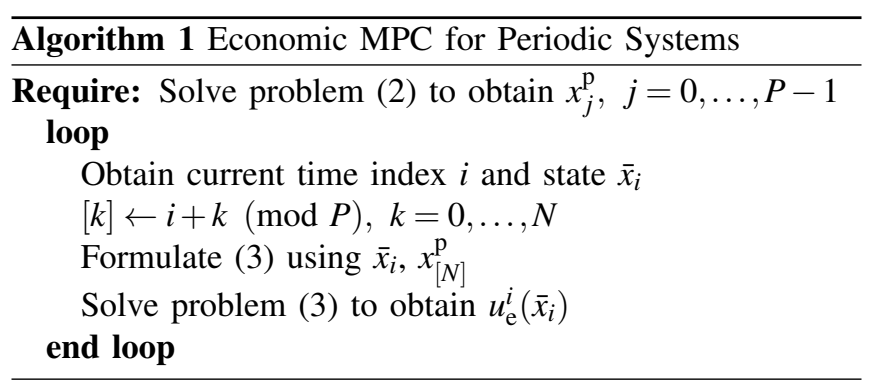

\section{NOMINAL StABILITY OF ECONOMIC MPC FOR PERIODIC SYSTEMS}

In this section, the asymptotic stability of the closed-loop system $\bar{x}_{i+1}=f_{[0]}\left(\bar{x}_{i}, u_{\mathrm{e}}^{i}\left(\bar{x}_{i}\right)\right)$ is established using the rotated cost in a form similar to [1]

$$
\begin{aligned}
L_{[k]}\left(x_{k}, u_{k}\right):= & l_{[k]}\left(x_{k}, u_{k}\right)-l_{[k]}\left(x_{[k]}^{\mathrm{p}}, u_{[k]}^{\mathrm{p}}\right)+\left(x_{k}-x_{[k]}^{\mathrm{p}}\right)^{T} \lambda_{[k]}^{\mathrm{p}} \\
& -\left(f_{[k]}\left(x_{k}, u_{k}\right)-f_{[k]}\left(x_{[k]}^{\mathrm{p}}, u_{[k]}^{\mathrm{p}}\right)\right)^{T} \lambda_{[k+1]}^{\mathrm{p}},
\end{aligned}
$$

and the Lyapunov function

$$
\tilde{V}_{N}^{[0]}\left(\bar{x}_{i}\right):=V_{N}^{[0]}\left(\bar{x}_{i}\right)+\left(\bar{x}_{i}-x_{[0]}^{\mathrm{p}}\right)^{T} \lambda_{[0]}^{\mathrm{p}}-\sum_{k=0}^{N-1} l_{[k]}\left(x_{[k]}^{\mathrm{p}}, u_{[k]}^{\mathrm{p}}\right) .
$$

Note that, according to $(1),[0]=i(\bmod P)$ holds, as displayed in Figure 1.

In order to prove this result, the following definitions and assumptions are needed.

Definition 1 (Feasible Control Sequence): A control sequence $\mathbf{u}=\left(u_{0}, \ldots, u_{N-1}\right)$ is termed feasible if the trajectory $\left(x_{0}, u_{0}, \ldots, x_{N}\right)$ satisfies the constraints of optimization problem (3).

Definition 2 (Admissible Set and Admissible States): At time $i$, the admissible set $\mathbb{Z}_{N}^{i}$ is defined as the set of all feasible trajectories, i.e.:

$$
\begin{aligned}
\mathbb{Z}_{N}^{i}=\left\{\left(\bar{x}_{i}, \mathbf{u}\right) \mid\right. & \exists x_{1}, \ldots, x_{N}: x_{0}=\bar{x}_{i}, x_{k+1}=f_{[k]}\left(x_{k}, u_{k}\right), \\
& \left.g_{[k]}\left(x_{k}, u_{k}\right) \leq 0, k=0, \ldots, N-1, x_{N}=x_{[N]}^{\mathrm{p}}\right\} .
\end{aligned}
$$

and the set $X_{N}^{i}$ is defined as the corresponding set of admissible states, i.e.:

$$
X_{N}^{i}=\left\{\bar{x}_{i} \mid \exists \mathbf{u}:\left(\bar{x}_{i}, \mathbf{u}\right) \in \mathbb{Z}_{N}^{i}\right\} .
$$

Assumption 1 (Lipschitz Continuity of $f$ and $l$ ): In the following, the Lipschitz continuity of $f(\cdot)$ and $l .(\cdot)$ on the admissible set $\mathbb{Z}_{N}^{i}$ is assumed, i.e. there exist $L_{f}, L_{l}>0$ such that

$$
\begin{aligned}
\left\|f_{j}(x, u)-f_{j}(\hat{x}, \hat{u})\right\| & \leq L_{f}\|(x, u)-(\hat{x}, \hat{u})\| \\
\left\|l_{j}(x, u)-l_{j}(\hat{x}, \hat{u})\right\| & \leq L_{l}\|(x, u)-(\hat{x}, \hat{u})\|
\end{aligned}, j=0, \ldots, P-1,
$$

where $\|$.$\| is any appropriate norm.$ 
The dynamic system is required to have some form of controllability, given by the following assumption.

Assumption 2 (Weak Controllability): There exists a $K_{\infty}$ function [6] $\gamma(\cdot)$ such that for every $\bar{x}_{i} \in X_{N}^{i}$ there exists u such that $\left(\bar{x}_{i}, \mathbf{u}\right) \in \mathbb{Z}_{N}^{i}$ and

$$
\sum_{k=0}^{N-1}\left\|u_{k}-u_{[k]}^{\mathrm{p}}\right\| \leq \gamma\left(\left\|\bar{x}_{i}-x_{[0]}^{\mathrm{p}}\right\|\right) .
$$

This assumption is also used in [4] and it bounds the cost of steering the system from an admissible initial state $\bar{x}_{i}$ to $x_{[N]}^{\mathrm{p}}$ in $N$ steps while satisfying the state and control constraints.

Additionally, strong duality of the periodic optimization problem (2) is required, i.e.

Assumption 3 (Strong Duality of the Periodic Problem): There exists a series of multipliers $\lambda_{j}^{\mathrm{p}}$ so that the trajectory $\left(x_{j}^{\mathrm{p}}, u_{j}^{\mathrm{p}}\right), j=0, \ldots, P-1$ uniquely solves

$$
\begin{aligned}
\min _{x_{0}, u_{0}, \ldots, x_{P-1}} & \sum_{j=0}^{P-1} l_{j}\left(x_{j}, u_{j}\right)+\sum_{j=0}^{P-2}\left(x_{j+1}-f_{j}\left(x_{j}, u_{j}\right)\right)^{T} \lambda_{j+1}^{\mathrm{p}} \\
& +\left(x_{0}-f_{0}\left(x_{P-1}, u_{P-1}\right)\right)^{T} \lambda_{0}^{\mathrm{p}} \\
& g_{j}\left(x_{j}, u_{j}\right) \leq 0, \quad \forall j=0, \ldots, P-1 .
\end{aligned}
$$

Moreover, there exists a $K_{\infty}$-function $\beta(\cdot)$ such that the "rotated" stage cost function (5) satisfies

$$
L_{j}(x, u) \geq \beta\left(\left\|x-x_{j}^{\mathrm{p}}\right\|\right),
$$

for all $(x, u)$ satisfying $g_{j}(x, u) \leq 0$. Note that if (8) holds, (7) is implied.

This assumption holds for strictly convex problems, which include periodic optimal control of linear time-varying (LTV) systems subject to convex constraints $g_{j}(x, u) \leq 0$. This assumption could be replaced by a generalization of the dissipativity assumption proposed in [3] to the periodic case. Yet, for the sake of simplicity, the more restrictive Assumption 3 is used in this paper. The dissipativity assumption is being considered in current work on the stability of E-NMPC.

Theorem 1: If Assumptions 2 and 3 hold, the solution of the closed-loop system $\bar{x}_{i+1}=f_{[0]}\left(\bar{x}_{i}, u_{\mathrm{e}}^{i}\left(\bar{x}_{i}\right)\right)$ is asymptotically stable with $X_{N}^{i}$ as the region of attraction, and admits $\tilde{V}_{N}^{[0]}\left(\bar{x}_{i}\right)$ (see (5)) as a Lyapunov function, i.e. $\tilde{V}_{N}^{[1]}\left(\bar{x}_{i+1}\right) \leq$ $\tilde{V}_{N}^{[0]}\left(\bar{x}_{i}\right)-L_{[0]}\left(\bar{x}_{i}, u_{\mathrm{e}}^{i}\left(\bar{x}_{i}\right)\right)$.

Proof: The proof is based on the following two lemmas. The first one compares the MPC scheme (3) with a "rotated" MPC scheme, that uses $L .(\cdot, \cdot)$ as stage cost. The second one shows that the value $\tilde{V}_{N}^{[0]}\left(\bar{x}_{i}\right)$ of the rotated MPC scheme is a Lyapunov function for the closed-loop system. The rotated
MPC scheme is defined as

$$
\begin{aligned}
\tilde{V}_{N}^{[0]}\left(\bar{x}_{i}\right):= & \\
\min _{x_{0}, u_{0}, \ldots, x_{N}} & \sum_{k=0}^{N-1} L_{[k]}\left(x_{k}, u_{k}\right) \\
\text { s.t. } \quad & x_{0}-\bar{x}_{i}=0 \\
& x_{k+1}-f_{[k]}\left(x_{k}, u_{k}\right)=0, \quad k=0, \ldots, N-1 \\
& g_{[k]}\left(x_{k}, u_{k}\right) \leq 0, \quad k=0, \ldots, N-1 \\
& x_{N}-x_{[N]}^{\mathrm{p}}=0 .
\end{aligned}
$$

Lemma 1: The solutions of problems (3) and (9) coincide and $\tilde{V}_{N}^{[0]}\left(\bar{x}_{i}\right)=V_{N}^{[0]}\left(\bar{x}_{i}\right)+\left(\bar{x}_{i}-x_{[0]}^{\mathrm{p}}\right)^{T} \lambda_{[0]}^{\mathrm{p}}-\sum_{k=0}^{N-1} l_{[k]}\left(x_{[k]}^{\mathrm{p}}, u_{[k]}^{\mathrm{p}}\right)$ holds.

Proof: Observing that problems (3) and (9) are subject to the same constraints, the objective function of (9) can be transformed as follows. One can add the constant term $l_{[k]}\left(x_{[k]}^{\mathrm{p}}, u_{[k]}^{\mathrm{p}}\right)$ to obtain

$$
\begin{aligned}
& \sum_{k=0}^{N-1} L_{[k]}\left(x_{k}, u_{k}\right)+l_{[k]}\left(x_{[k]}^{\mathrm{p}}, u_{[k]}^{\mathrm{p}}\right) \\
& =\sum_{k=0}^{N-1} l_{[k]}\left(x_{k}, u_{k}\right)+\left(x_{k}-x_{[k]}^{\mathrm{p}}\right)^{T} \lambda_{[k]}^{\mathrm{p}} \\
& \quad-\left(f_{[k]}\left(x_{k}, u_{k}\right)-f_{[k]}\left(x_{[k]}^{\mathrm{p}}, u_{[k]}^{\mathrm{p}}\right)\right)^{T} \lambda_{[k+1]}^{\mathrm{p}} .
\end{aligned}
$$

Then, adding and subtracting the term $\left(x_{k+1}-x_{[k+1]}^{\mathrm{p}}\right)^{T} \lambda_{[k+1]}^{\mathrm{p}}$, Equation (10) becomes

$$
\begin{aligned}
& \quad \sum_{k=0}^{N-1} L_{[k]}\left(x_{k}, u_{k}\right)+l_{[k]}\left(x_{[k]}^{\mathrm{p}}, u_{[k]}^{\mathrm{p}}\right) \\
& =\sum_{k=0}^{N-1} l_{[k]}\left(x_{k}, u_{k}\right)+\left(x_{k}-x_{[k]}^{\mathrm{p}}\right)^{T} \lambda_{[k]}^{\mathrm{p}} \\
& \quad-\left(f_{[k]}\left(x_{k}, u_{k}\right)-f_{[k]}\left(x_{[k]}^{\mathrm{p}}, u_{[k]}^{\mathrm{p}}\right)\right)^{T} \lambda_{[k+1]}^{\mathrm{p}} \\
& \quad+\left(x_{k+1}-x_{[k+1]}^{\mathrm{p}}\right)^{T} \lambda_{[k+1]}^{\mathrm{p}}-\left(x_{k+1}-x_{[k+1]}^{\mathrm{p}}\right)^{T} \lambda_{[k+1]}^{\mathrm{p}} .
\end{aligned}
$$

The terms $\left(x_{k}-x_{[k]}^{\mathrm{p}}\right)^{T} \lambda_{[k]}^{\mathrm{p}}, k=1, \ldots, N-1$ and $-\left(x_{k+1}-\right.$ $\left.x_{[k+1]}^{\mathrm{p}}\right)^{T} \lambda_{[k+1]}^{\mathrm{p}}, k=0, \ldots, N-2$ cancel out. Moreover, because of the dynamic constraints (9c) and (2b)-(2c), for any $\left(\bar{x}_{i}, \mathbf{u}\right) \in \mathbb{Z}_{N}^{i}$, it holds that $\left(x_{k+1}-x_{[k+1]}^{\mathrm{p}}\right)-\left(f_{[k]}\left(x_{k}, u_{k}\right)-\right.$ $\left.f_{[k]}\left(x_{[k]}^{\mathrm{p}}, u_{[k]}^{\mathrm{p}}\right)\right)=0$. Finally, $\left(x_{N}-x_{[N]}^{\mathrm{p}}\right)=0$ holds due to $(9 \mathrm{e})$.

Equation (10) thus reads:

$$
\begin{aligned}
\sum_{k=0}^{N-1} L_{[k]}\left(x_{k}, u_{k}\right) & +l_{[k]}\left(x_{[k]}^{\mathrm{p}}, u_{[k]}^{\mathrm{p}}\right) \\
& =\sum_{k=0}^{N-1} l_{[k]}\left(x_{k}, u_{k}\right)+\left(x_{0}-x_{[0]}^{\mathrm{p}}\right)^{T} \lambda_{[0]}^{\mathrm{p}} .
\end{aligned}
$$

From $(9 \mathrm{~b}),\left(x_{0}-\bar{x}_{i}\right)=0$ holds. Then, adding and subtract- 


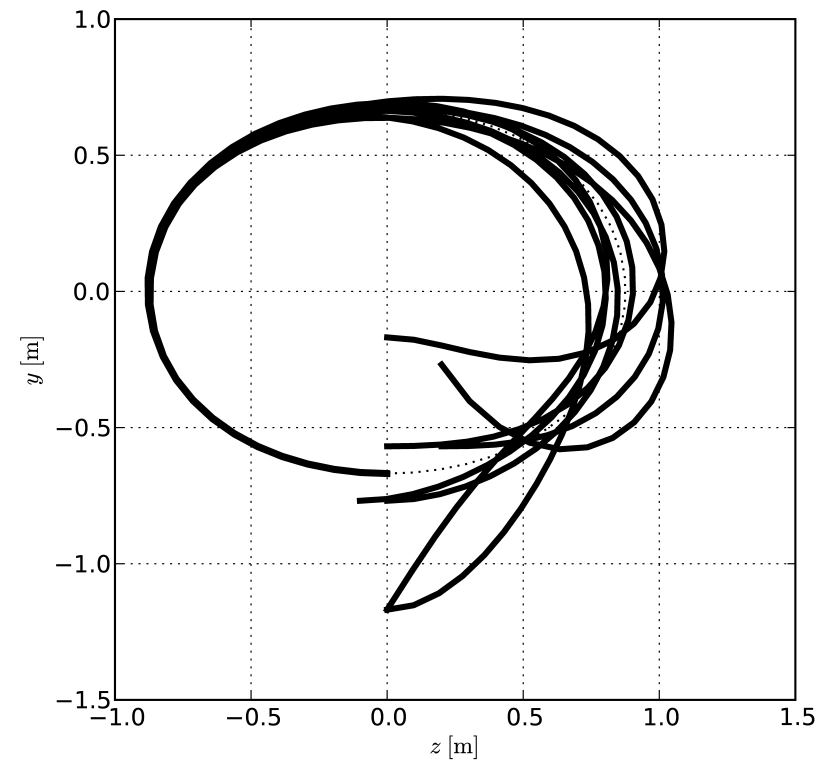

Fig. 2. The trajectories obtained by applying the economic MPC scheme to the system starting from different initial conditions are displayed in thick line. The system is steered to the periodic trajectory solution of the periodic optimal control problem (2) (thin line).

ing the term $\bar{x}_{i}^{T} \lambda_{[0]}^{\mathrm{p}}$ to (11), one finally obtains:

$$
\begin{aligned}
\sum_{k=0}^{N-1} L_{[k]}\left(x_{k}, u_{k}\right) & +l_{[k]}\left(x_{[k]}^{\mathrm{p}}, u_{[k]}^{\mathrm{p}}\right) \\
& =\sum_{k=0}^{N-1} l_{[k]}\left(x_{k}, u_{k}\right)+\left(\bar{x}_{i}-x_{[0]}^{\mathrm{p}}\right)^{T} \lambda_{[0]}^{\mathrm{p}},
\end{aligned}
$$

The rotated cost thus differs from the original one only by a constant term and the solutions to problems (3) and (9) are identical.

Taking $P=1$, (2) yields a steady-state trajectory, and Lemma 1 entails the result obtained in [1].

Lemma 2: For all $\bar{x}_{i} \in X_{N}^{i}$, the value $\tilde{V}_{N}^{[0]}\left(\bar{x}_{i}\right)$ of the rotated MPC scheme is a Lyapunov function, i.e. there are two $K_{\infty}$ functions $\alpha(\cdot)$ and $\beta(\cdot)$ such that

$$
\beta\left(\left\|\bar{x}_{i}-x_{[0]}^{\mathrm{p}}\right\|\right) \leq \tilde{V}_{N}^{[0]}\left(\bar{x}_{i}\right) \leq \alpha\left(\left\|\bar{x}_{i}-x_{[0]}^{\mathrm{p}}\right\|\right),
$$

and it holds that (descent condition):

$$
\begin{aligned}
\tilde{V}_{N}^{[1]}\left(f_{[0]}\left(\bar{x}_{i}, u_{\mathrm{e}}^{i}\left(\bar{x}_{i}\right)\right)\right)-\tilde{V}_{N}^{[0]}\left(\bar{x}_{i}\right) & \leq-L_{[0]}\left(\bar{x}_{i}, u_{\mathrm{e}}^{i}\left(\bar{x}_{i}\right)\right) \\
& \leq-\beta\left(\left\|\bar{x}_{i}-x_{[0]}^{\mathrm{p}}\right\|\right) .
\end{aligned}
$$

Proof: The proof is given in the appendix; it is an extension of the one provided in [1] and follows similar arguments.

\section{APPLiCATION EXAMPLES}

In this section, two simple examples will be used to illustrate the proposed method. First, a simple car model will be considered. The system dynamics are given by

$$
\left[\begin{array}{c}
\dot{z} \\
\dot{y} \\
\dot{\theta}
\end{array}\right]=\left[\begin{array}{c}
v \cos \theta \\
v \sin \theta \\
u
\end{array}\right],
$$
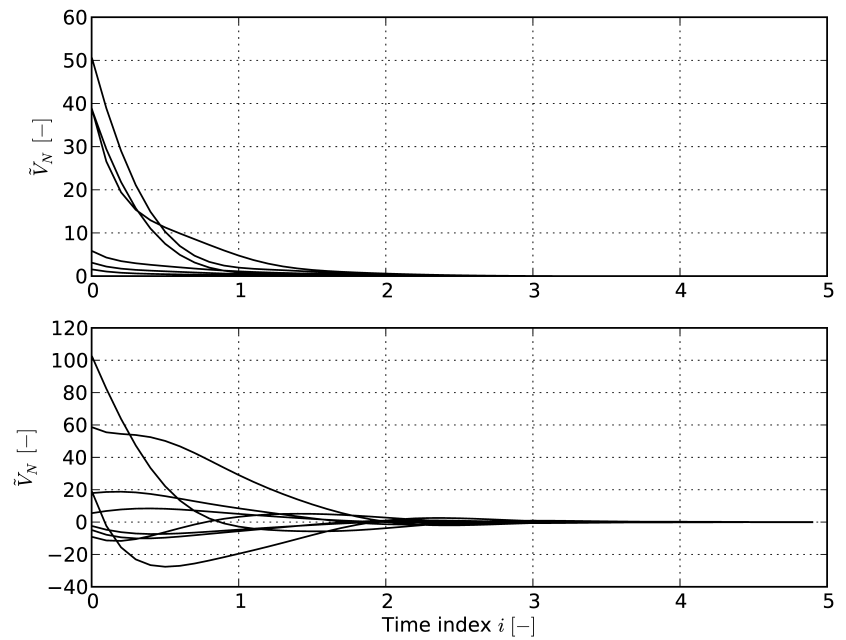

Fig. 3. Rotated cost $\tilde{V}_{N}^{[0]}$ over time. In the top graph, the rotated cost obtained with the proposed framework monotonically decreases and is a Lyapunov function. In the bottom graph, the cost rotated as proposed in [4] is not a Lyapunov function for the given example.

where the position is given by $(z, y)$, the angle $\theta$ indicates the orientation of the vehicle, the control $u$ is a rotational velocity and the vehicle has constant linear velocity $v=1 \mathrm{~m} / \mathrm{s}$.

All optimization problems have been solved using the direct multiple shooting [7] method. The system dynamics have been translated into discrete time using a fixed stepsize Runge-Kutta integrator of order 4, with a sampling time $T_{\mathrm{s}}=0.1 \mathrm{~s}$ and a piecewise constant control parametrization. The period has been chosen as $P=50$. The problems have been formulated using the open source software CasADi [8] and the NLP solver Ipopt [9].

Given the stage cost $l_{j}\left(x_{j}, u_{j}\right)=u_{j}^{2}+z_{j}^{2}+5 y_{j}^{2}$ and the periodicity conditions, the solution of problem (2) yields a periodic trajectory. Since for this periodic optimization problem strong duality does not hold, for the MPC formulation, the Linear Time Varying (LTV) model is considered here. The LTV model is obtained by linearizing the integrator on the optimal periodic trajectory.

Starting from an initial state $\left(z_{\text {init }}, y_{\text {init }}, \theta_{\text {init }}\right)$, at each time instant $i$, problem (3) is solved following Algorithm 1 for a horizon length $N=20$. The obtained trajectory is shown in Figure 2, where it can be noted that the system is stabilized to the optimal periodic trajectory. Figure 3 displays the value function $\tilde{V}_{N}^{[0]}\left(\bar{x}_{i}\right)$ at each time instant in the top graph. It satisfies the requirements of a Lyapunov function as it is bounded from below by a $K_{\infty}$-function and decreases monotonically. As a term of comparison, the approach proposed in [4] has also been implemented and the obtained value function is displayed in Figure 3, bottom graph. It can be seen that this function does not monotonically decrease and becomes negative.

As a second illustrative example, the double-tank system proposed in [4] was also treated. The proposed scheme stabilizes the system and the value function $\tilde{V}_{N}^{[0]}\left(\bar{x}_{i}\right)$ is positive and monotonically decreasing (see Figure 4). For 

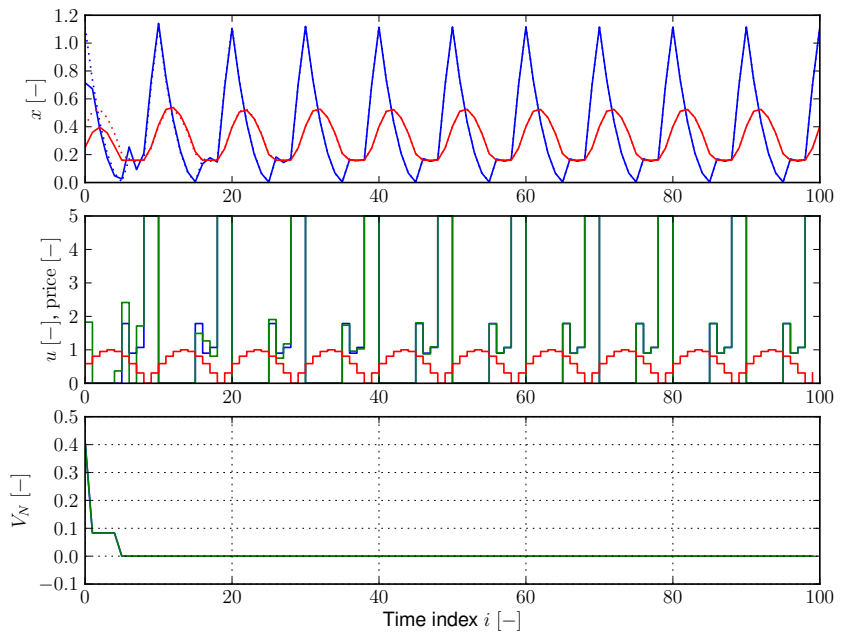

Fig. 4. In the top graph: plant state in thick line, optimal periodic state in dotted line. Middle graph: applied controls (green), optimal periodic controls (blue) and price (red). Bottom graph: rotated cost $\tilde{V}_{N}^{[0]}$ over time. In this example the rotated cost $\tilde{V}_{N}^{[0]}$ (blue line) coincides with the cost rotated as proposed in [4] (green line).

this example, the rotated cost proposed in [4] coincides with the one defined here.

The stability proof established in this paper relies on the strong duality Assumption 3. This assumption is not verified for the car example if the nonlinear model is used instead of the LTV one. By applying the nonlinear MPC scheme, one obtains trajectories that differ slightly from the ones of the LTV model. Those trajectories are displayed in Figure 5. While the MPC scheme still successfully stabilizes the plant, the rotated cost is not a Lyapunov function for the system, as for some initial conditions it does not monotonically decrease (see Figure 6). Further investigation of the stability of economic MPC schemes for which Assumption 3 is not verified is the subject of ongoing research.

\section{CONCLUSION}

In this paper, a Lyapunov function was proposed for the class of problems having a periodic optimal trajectory and satisfying a strong duality assumption. This class of problems includes linear time-varying systems subject to convex constraints and strictly convex cost.

The proposed theory has been illustrated with two numerical examples and is a generalization of the results proposed in [1].

Future work will focus on the extension of the presented results to more general classes of dynamical systems and for the case of relaxed or no terminal constraints. The use of a dissipativity assumption is currently being investigated for this purpose.

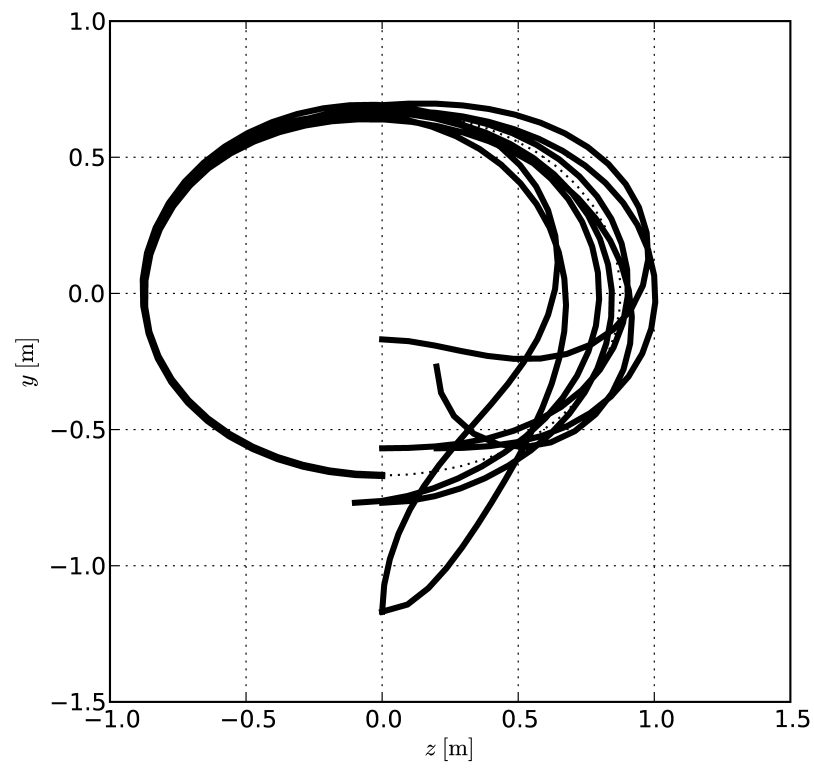

Fig. 5. The trajectories obtained by applying the nonlinear economic MPC scheme to the system starting from different initial conditions are displayed in thick line. The system is steered to the periodic trajectory solution of the periodic optimal control problem (2) (thin line).
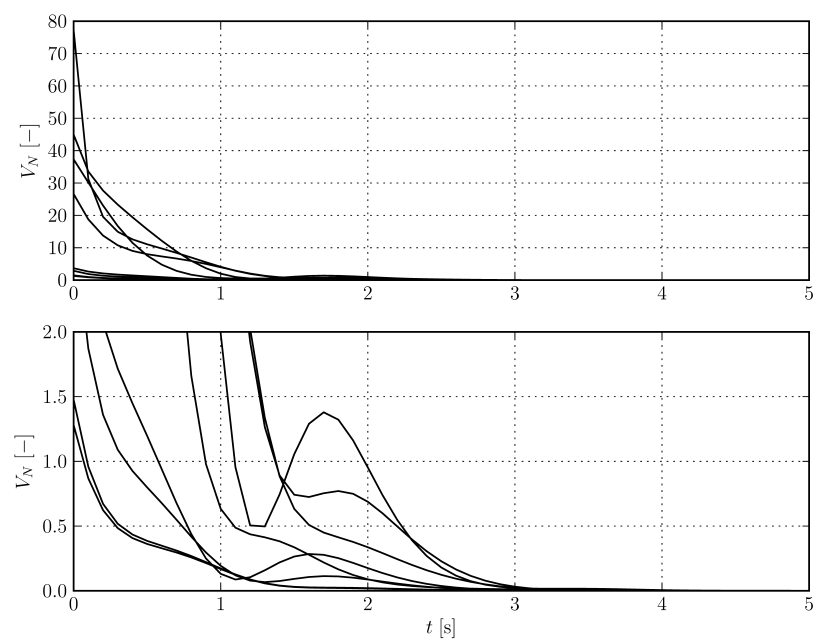

Fig. 6. Rotated cost $\tilde{V}_{N}^{[0]}$ over time. In the bottom graph, a closer zoom where it can be seen that the rotated cost is not a Lyapunov function.

\section{APPENDIX}

This section provides the proof of Lemma 2, which states the existence of two $K_{\infty}$-functions $\alpha(\cdot)$ and $\beta(\cdot)$ such that

$$
\begin{array}{r}
\beta\left(\left\|\bar{x}_{i}-x_{[0]}^{\mathrm{p}}\right\|\right) \leq \tilde{V}_{N}^{[0]}\left(\bar{x}_{i}\right) \leq \alpha\left(\left\|\bar{x}_{i}-x_{[0]}^{\mathrm{p}}\right\|\right), \\
\tilde{V}_{N}^{[1]}\left(f_{[0]}\left(\bar{x}_{i}, u_{\mathrm{e}}^{i}\left(\bar{x}_{i}\right)\right)\right)-\tilde{V}_{N}^{[0]}\left(\bar{x}_{i}\right) \leq-\beta\left(\left\|\bar{x}_{i}-x_{[0]}^{\mathrm{p}}\right\|\right) .
\end{array}
$$

The proof is an extension of the one provided in [1] and follows similar arguments. The left inequality in (15) and the descent condition (16) are proven first.

Lemma 3: The function $\tilde{V}_{N}^{[0]}\left(\bar{x}_{i}\right)$ is bounded from below 
by

$$
\tilde{V}_{N}^{[0]}\left(\bar{x}_{i}\right) \geq \beta\left(\left\|\bar{x}_{i}-x_{[0]}^{\mathrm{p}}\right\|\right),
$$

and

$$
\tilde{V}_{N}^{[1]}\left(f_{[0]}\left(\bar{x}_{i}, u_{\mathrm{e}}^{i}\left(\bar{x}_{i}\right)\right)\right)-\tilde{V}_{N}^{[0]}\left(\bar{x}_{i}\right) \leq-\beta\left(\left\|\bar{x}_{i}-x_{[0]}^{\mathrm{p}}\right\|\right) .
$$

Proof: Starting from a point $\bar{x}_{i}$ and solving problem (9), by applying the optimal control policy $u_{\mathrm{e}}^{i}\left(\bar{x}_{i}\right)$ to the system, one obtains

$$
\tilde{V}_{N-1}^{[1]}\left(f_{[0]}\left(\bar{x}_{i}, u_{\mathrm{e}}^{i}\left(\bar{x}_{i}\right)\right)\right)=\tilde{V}_{N}^{[0]}\left(\bar{x}_{i}\right)-L_{[0]}\left(\bar{x}_{i}, u_{\mathrm{e}}^{i}\left(\bar{x}_{i}\right)\right) .
$$

The trajectory obtained by eliminating the first state from the optimal one and prolonging it by applying the control $u_{[N]}^{\mathrm{p}}$, is a feasible trajectory. The cost associated to this trajectory is given by $\tilde{V}_{N-1}^{[1]}\left(f_{[0]}\left(\bar{x}_{i}, u_{\mathrm{e}}^{i}\left(\bar{x}_{i}\right)\right)\right)+L_{[N]}\left(x_{[N]}^{\mathrm{p}}, u_{[N]}^{\mathrm{p}}\right)$. By definition $L_{N}\left(x_{[N]}^{\mathrm{p}}, u_{[N]}^{\mathrm{p}}\right)=0$, thus optimality implies that

$$
\tilde{V}_{N}^{[1]}\left(f_{[0]}\left(\bar{x}_{i}, u_{\mathrm{e}}^{i}\left(\bar{x}_{i}\right)\right)\right) \leq \tilde{V}_{N}^{[0]}\left(\bar{x}_{i}\right)-L_{[0]}\left(\bar{x}_{i}, u_{\mathrm{e}}^{i}\left(\bar{x}_{i}\right)\right) .
$$

Using Assumption 3, one obtains

$$
\begin{aligned}
\tilde{V}_{N}^{[1]}\left(f_{[0]}\left(\bar{x}_{i}, u_{\mathrm{e}}^{i}\left(\bar{x}_{i}\right)\right)\right)-\tilde{V}_{N}^{[0]}\left(\bar{x}_{i}\right) & \leq-L_{[0]}\left(\bar{x}_{i}, u_{\mathrm{e}}^{i}\left(\bar{x}_{i}\right)\right) \\
& \leq-\beta\left(\left\|\bar{x}_{i}-x_{[0]}^{\mathrm{p}}\right\|\right),
\end{aligned}
$$

and the result is established.

For proving the right inequality of Equation (15), let the sub-optimal rotated cost function be defined as

$$
\tilde{V}_{N}^{[0]}\left(\bar{x}_{i}, \mathbf{u}\right)=\left(\bar{x}_{i}-x_{[0]}^{\mathrm{p}}\right)^{T} \lambda_{[0]}^{\mathrm{p}}+\sum_{k=0}^{N-1} l_{[k]}\left(x_{k}, u_{k}\right)-l_{[k]}\left(x_{[k]}^{\mathrm{p}}, u_{[k]}^{\mathrm{p}}\right),
$$

which depends on the initial state $\bar{x}_{i}$ and the feasible control sequence $\mathbf{u}=\left(u_{0}, u_{1}, \ldots, u_{N-1}\right)$. Using the triangle inequality and Lipschitz continuity of $l \cdot(\cdot, \cdot)$ (Assumption 1) one can write

$\tilde{V}_{N}^{[0]}\left(\bar{x}_{i}, \mathbf{u}\right) \leq\left\|\bar{x}_{i}-x_{[0]}^{\mathrm{p}}\right\|\left\|\lambda_{[0]}^{\mathrm{p}}\right\|+\sum_{k=0}^{N-1} L_{l}\left\|x_{k}-x_{[k]}^{\mathrm{p}}\right\|+L_{l}\left\|u_{k}-u_{[k]}^{\mathrm{p}}\right\|$

By using the system equations $x_{k+1}=f_{[k]}\left(x_{k}, u_{k}\right)$ and the Lipschitz continuity of $f(\cdot, \cdot)$ (Assumption 1), it holds that for all $\left(\bar{x}_{i}, \mathbf{u}\right) \in \mathbb{Z}_{N}^{i}$ and $k \geq 0$

$$
\begin{aligned}
\left\|x_{k}-x_{[k]}^{\mathrm{p}}\right\| \leq L_{f}^{k}\left\|\bar{x}_{i}-x_{[0]}^{\mathrm{p}}\right\| & +L_{f}^{k}\left\|u_{0}-u_{[0]}^{\mathrm{p}}\right\|+L_{f}^{k-1}\left\|u_{1}-u_{[1]}^{\mathrm{p}}\right\| \\
& +\cdots+L_{f}\left\|u_{k-1}-u_{[k-1]}^{\mathrm{p}}\right\| .
\end{aligned}
$$

Summing these inequalities yields

$$
\sum_{k=0}^{N-1}\left\|x_{k}-x_{[k]}^{\mathrm{p}}\right\| \leq L_{F}\left\|\bar{x}_{i}-x_{[0]}^{\mathrm{p}}\right\|+L_{F} \sum_{k=0}^{N-1}\left\|u_{k}-u_{[k]}^{\mathrm{p}}\right\|,
$$

where $L_{F}=1+L_{f}+\cdots+L_{f}^{N-1}$.

The weak controllability Assumption 2 ensures that for all $\bar{x}_{i} \in X_{N}^{i}$, there exist $\left(\bar{x}_{i}, \mathbf{u}\right) \in \mathbb{Z}_{N}^{i}$ such that

$$
\sum_{k=0}^{N-1}\left\|u_{k}-u_{[k]}^{\mathrm{p}}\right\| \leq \gamma\left(\left\|\bar{x}_{i}-x_{[0]}^{\mathrm{p}}\right\|\right) \text {. }
$$

By using (21) and (22), one obtains that, for all $\left(\bar{x}_{i}, \mathbf{u}\right) \in \mathbb{Z}_{N}^{i}$, the following inequality holds

$$
\begin{aligned}
\sum_{k=0}^{N-1} L_{l}\left(\left\|x_{k}-x_{[k]}^{\mathrm{p}}\right\|+\left\|u_{k}-u_{[k]}^{\mathrm{p}}\right\|\right) & \leq L_{l} L_{F}\left\|\bar{x}_{i}-x_{[0]}^{\mathrm{p}}\right\| \\
& +L_{l}\left(1+L_{F}\right) \gamma\left(\left\|\bar{x}_{i}-x_{[0]}^{\mathrm{p}}\right\|\right) .
\end{aligned}
$$

Substituting this result into (20) and defining the $K_{\infty}$-function $\alpha(\cdot)=\left(\left\|\lambda_{[0]}^{\mathrm{p}}\right\|+L_{l} L_{F}\right)(\cdot)+L_{l}\left(1+L_{F}\right) \gamma(\cdot)$, yields

$$
\tilde{V}_{N}^{[0]}\left(\bar{x}_{i}, \mathbf{u}\right) \leq \alpha\left(\left\|\bar{x}_{i}-x_{[0]}^{\mathrm{p}}\right\|\right), \quad \forall\left(\bar{x}_{i}, \mathbf{u}\right) \in \mathbb{Z}_{N}^{i} .
$$

By optimizing over $\mathbf{u}$, one obtains the optimal value, which satisfies

$$
\tilde{V}_{N}^{[0]}\left(\bar{x}_{i}\right) \leq \alpha\left(\left\|\bar{x}_{i}-x_{[0]}^{\mathrm{p}}\right\|\right), \quad \forall \bar{x}_{i} \in X_{N}^{i},
$$

and the result is established.

\section{ACKNOWLEDGMENT}

This research was supported by Research Council KUL: PFV/10/002 Optimization in Engineering Center OPTEC, GOA/10/09 MaNet and GOA/10/11 Global real- time optimal control of autonomous robots and mechatronic systems. Flemish Government: IOF/KP/SCORES4CHEM, FWO: PhD/postdoc grants and projects: G.0320.08 (convex MPC), G.0377.09 (Mechatronics MPC); IWT: PhD Grants, projects: SBO LeCoPro; Belgian Federal Science Policy Office: IUAP P7 (DYSCO, Dynamical systems, control and optimization, 2012-2017); EU: FP7-EMBOCON (ICT-248940), FP7-SADCO ( MC ITN-264735), ERC ST HIGHWIND (259 166), Eurostars SMART, ACCM.

\section{REFERENCES}

[1] M. Diehl, R. Amrit, and J. Rawlings, "A Lyapunov Function for Economic Optimizing Model Predictive Control," IEEE Trans. of Automatic Control, vol. 56, pp. 703-707, March 2011.

[2] J. B. Rawlings and R. Amrit, "Optimizing Process Economic Performance using Model Predictive Control," in Proceedings of NMPC 08 Pavia, 2009.

[3] R. Amrit, J. Rawlings, and D. Angeli, "Economic optimization using model predictive control with a terminal cost," Annual Reviews in Control, 2011

[4] R. Huang, L. T. Biegler, and E. Harinath, "Robust stability of economically oriented infinite horizon NMPC that include cyclic processes," Journal of Process Control, vol. 22, pp. 51-29, 2012.

[5] L. Grüne, "NMPC Without Terminal Constraints," in Proceedings of the IFAC Conference on Nonlinear Model Predictive Control 2012, 2012.

[6] H. Khalil, Nonlinear Systems. Upper Saddle River, NJ: Prentice Hall second ed., 1996.

[7] H. Bock and K. Plitt, "A multiple shooting algorithm for direct solution of optimal control problems," in Proceedings 9th IFAC World Congress Budapest, pp. 242-247, Pergamon Press, 1984.

[8] J. Andersson, J. Åkesson, and M. Diehl, "CasADi - A symbolic package for automatic differentiation and optimal control," in Recent Advances in Algorithmic Differentiation (S. Forth, P. Hovland, E. Phipps, J. Utke, and A. Walther, eds.), Lecture Notes in Computational Science and Engineering, (Berlin), Springer, 2012.

[9] A. Wächter and L. Biegler, "On the Implementation of a Primal-Dual Interior Point Filter Line Search Algorithm for Large-Scale Nonlinear Programming," Mathematical Programming, vol. 106, no. 1, pp. 25-57, 2006. 